\title{
7-log virus removal in a simple functionalized sand filter
}

\author{
* Made by Ratul Chowdhury, Penn State. November 2. 2018. for PNAS
}

* This file contains CHARMM TOPOLOGY for GlcNAc molecule

220

MASS $201 \mathrm{OR} \quad 15.999400$

MASS $202 \mathrm{O}=\mathrm{C} \quad 15.999400$

MASS $203 \mathrm{NC}=\mathrm{O} 14.006700$

MASS $204 \mathrm{CR} \quad 12.011000$

MASS $205 \mathrm{C}=\mathrm{O} \quad 12.011000$

MASS 206 HCMM 1.007940

MASS $207 \mathrm{HNCO} 1.007940$

MASS 208 HOR 1.007940

AUTOGENERATE ANGLES DIHE

DEFA FIRS NONE LAST NONE

RESI LIG -0.000

GROUP

ATOM O OR $\quad-0.5600$

$\begin{array}{lll}\text { ATOM O1 OR } & -0.6800\end{array}$

ATOM O2 OR -0.6800

ATOM O3 OR -0.6800

ATOM O4 OR $\quad-0.6800$

ATOM O5 $\mathrm{O}=\mathrm{C}-0.5700$

ATOM N NC=O -0.7301

ATOM C CR 0.2800 
ATOM C1 CR 0.3001

ATOM C2 CR 0.2800

ATOM C3 CR 0.2800

ATOM C4 CR 0.5600

ATOM C5 CR 0.2800

ATOM C6 $\mathrm{C}=\mathrm{O} \quad 0.5690$

ATOM C7 CR 0.0610

ATOM H HCMM 0.0000

ATOM H1 HCMM 0.0000

ATOM H2 HCMM 0.0000

ATOM H3 HCMM 0.0000

ATOM H4 HCMM 0.0000

ATOM H5 HCMM 0.0000

ATOM H6 HCMM 0.0000

ATOM H7 HNCO 0.3700

ATOM H8 HOR 0.4000

ATOM H9 HOR 0.4000

ATOM H10 HOR 0.4000

ATOM H11 HOR 0.4000

ATOM H12 HCMM 0.0000

ATOM H13 HCMM 0.0000

ATOM H14 HCMM 0.0000

BOND O C3

BOND O C4

BOND O1 C

BOND O1 H8

BOND O2 C2

BOND O2 H9

BOND O3 C4

BOND O3 H10 


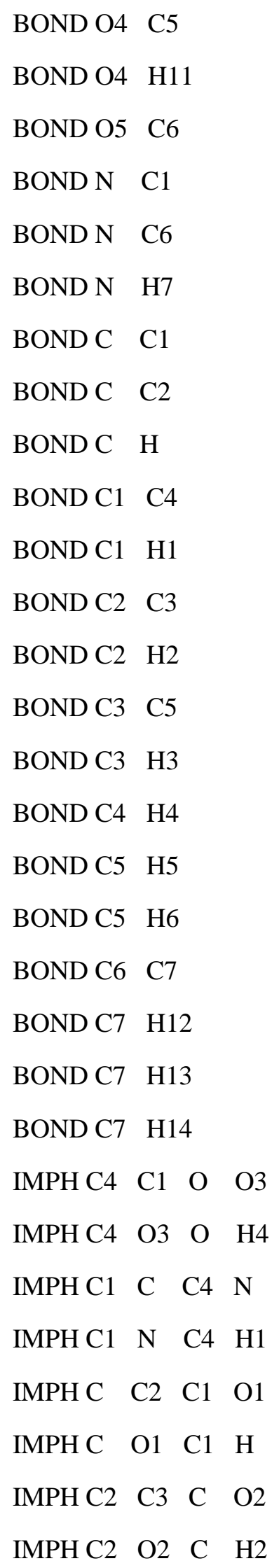




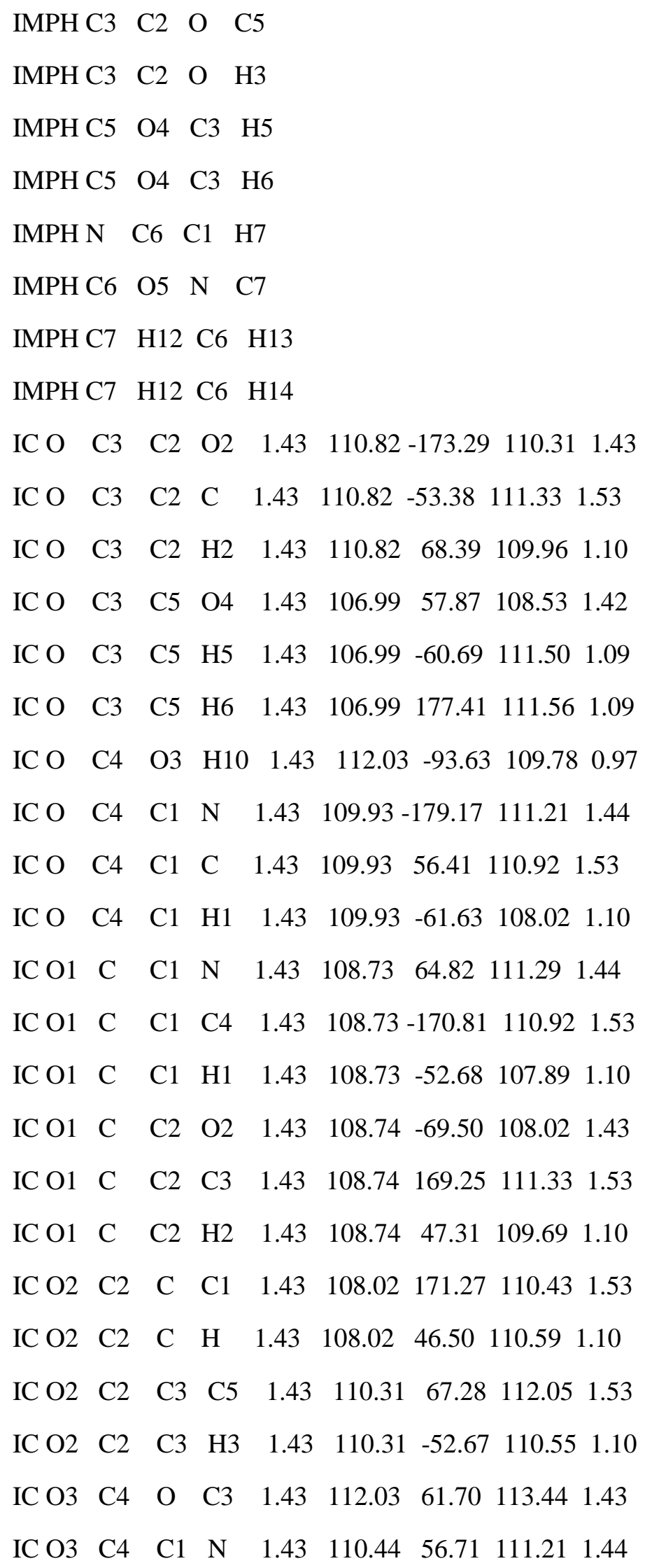




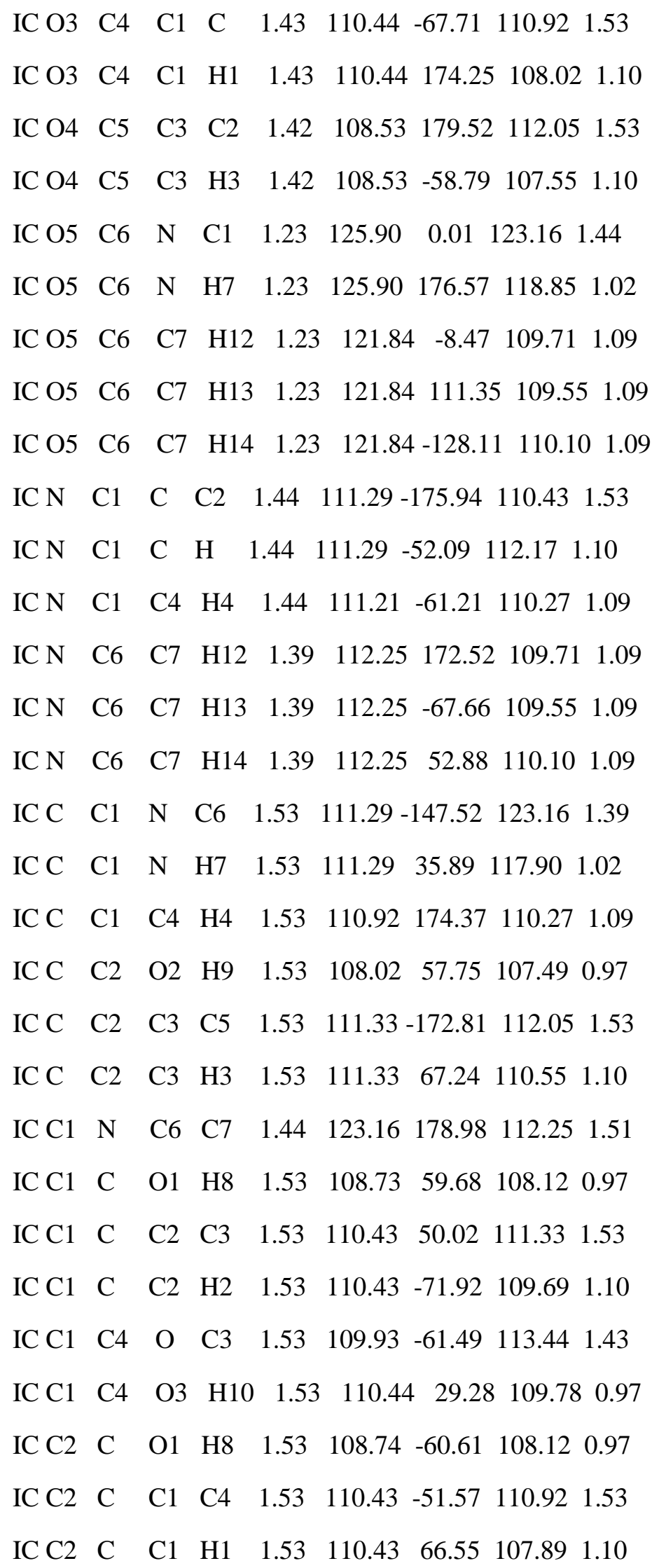




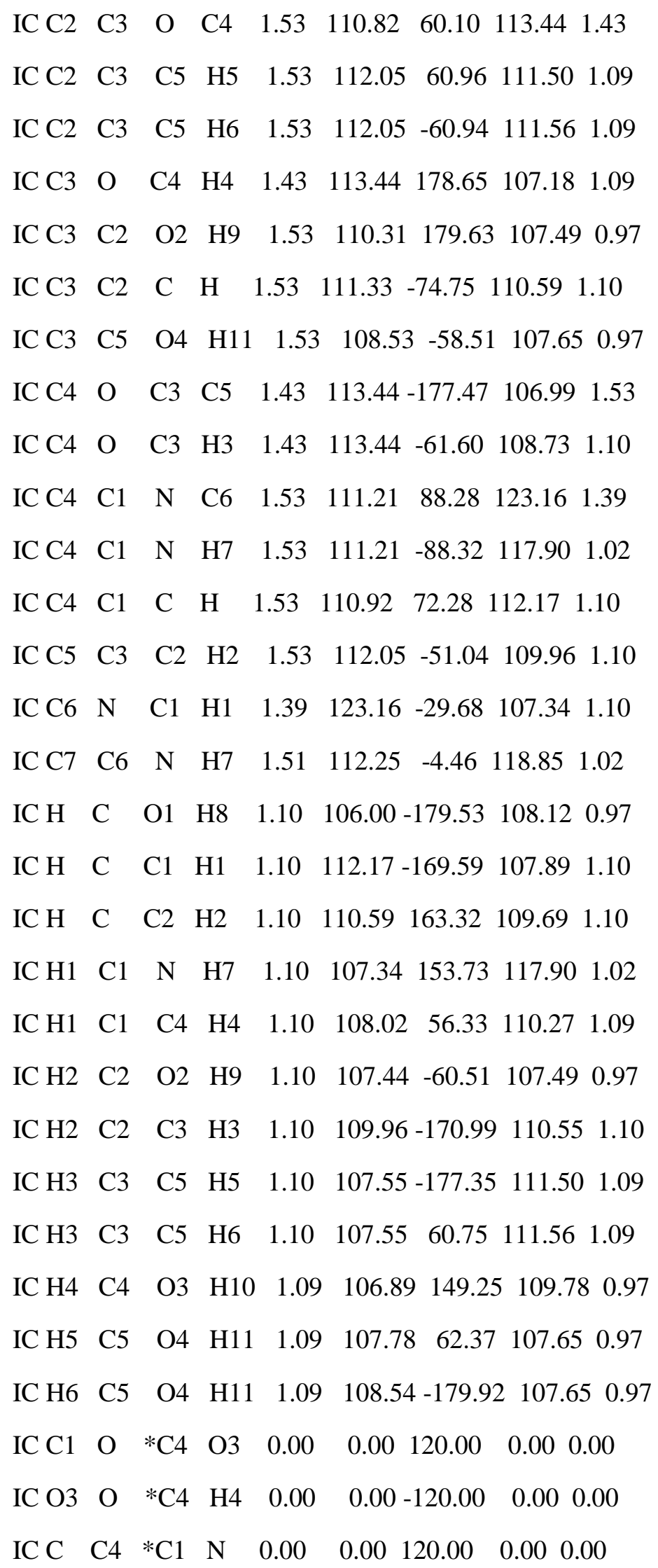




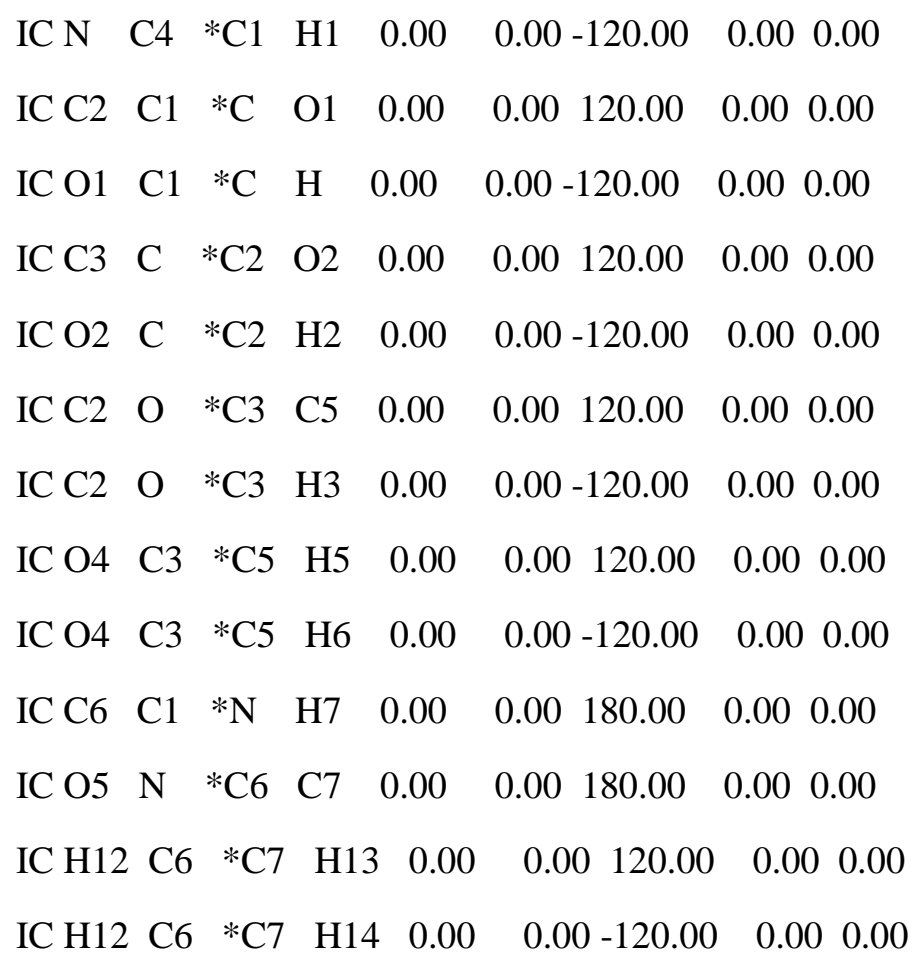




\author{
ANGLES \\ $\begin{array}{llll}\text { CR OR CR } & 86.143 \quad 106.9260\end{array}$ \\ CR OR HOR $57.069 \quad 106.5030$ \\ $\mathrm{CR} \quad \mathrm{NC}=\mathrm{O} \mathrm{C}=\mathrm{O} \quad 59.084 \quad 119.6000$ \\ CR $\quad \mathrm{NC}=\mathrm{O}$ HNCO $39.725 \quad 120.0660$ \\ $\mathrm{C}=\mathrm{O} \quad \mathrm{NC}=\mathrm{O}$ HNCO $\quad 41.380 \quad 120.2770$ \\ OR CR CR $71.390 \quad 108.1330$ \\ OR CR HCMM 56.205 108.5770 \\ $\begin{array}{llll}\text { CR CR CR } & 61.243 \quad 109.6080\end{array}$ \\ CR CR HCMM $45.770 \quad 110.5490$ \\ $\mathrm{NC}=\mathrm{O}$ CR $\quad \mathrm{CR} \quad 75.564 \quad 109.9600$ \\ $\mathrm{NC}=\mathrm{O}$ CR $\quad \mathrm{HCMM} \quad 53.255 \quad 107.6460$ \\ OR CR OR $83.192 \quad 111.3680$ \\ HCMM CR HCMM $37.134 \quad 108.8360$ \\ $\mathrm{O}=\mathrm{C} \quad \mathrm{C}=\mathrm{O} \quad \mathrm{NC}=\mathrm{O} \quad 65.273 \quad 127.1520$ \\ $\mathrm{O}=\mathrm{C} \quad \mathrm{C}=\mathrm{O} \quad \mathrm{CR} \quad 67.504 \quad 124.4100$ \\ $\mathrm{NC}=\mathrm{O} \mathrm{C}=\mathrm{O} \quad \mathrm{CR} \quad 70.814 \quad 112.7350$ \\ $\mathrm{C}=\mathrm{O} \quad \mathrm{CR} \quad \mathrm{HCMM} \quad 46.778 \quad 108.3850$
}

\title{
DIHEDRALS
}

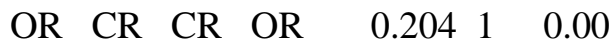

$\begin{array}{llllll}\text { OR CR CR OR } & 0.699 & 2 & 180.00\end{array}$

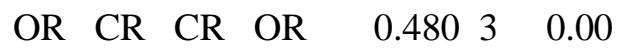

$\begin{array}{llllll}\text { OR CR CR } & \text { CR } & -0.344 & 1 & 0.00\end{array}$

$\begin{array}{llllll}\text { OR CR CR CR } & 0.879 & 2 & 180.00\end{array}$

$\begin{array}{llllll}\text { OR CR CR CR } & 0.238 & 3 & 0.00\end{array}$

$\begin{array}{llllll}\text { OR CR CR HCMM } & -0.327 & 1 & 0.00\end{array}$

$\begin{array}{llllll}\text { OR CR CR HCMM } & 0.536 & 2 & 180.00\end{array}$

$\begin{array}{lllllll}\text { OR CR CR HCMM } & 0.140 & 3 & 0.00\end{array}$

$\begin{array}{lllllll}\text { OR CR OR HOR } & 0.744 & 1 & 0.00\end{array}$ 
$\begin{array}{llllll}\text { OR CR OR HOR } & -1.700 & 2 & 180.00\end{array}$

$\begin{array}{llllll}\text { OR CR OR HOR } & -0.160 & 3 & 0.00\end{array}$

OR CR CR NC=O $0.150 \quad 3 \quad 0.00$

$\begin{array}{lllllll}\text { OR } & \text { CR } & \text { OR } & \text { CR } & 0.115 & 1 & 0.00\end{array}$

$\begin{array}{lllll}\text { OR CR OR CR } & -0.355 & 2 & 180.00\end{array}$

$\begin{array}{llllll}\text { OR CR OR CR } & 0.361 & 3 & 0.00\end{array}$

$\mathrm{O}=\mathrm{C} \quad \mathrm{C}=\mathrm{O} \quad \mathrm{NC}=\mathrm{O} \mathrm{CR} \quad-0.160 \quad 1 \quad 0.00$

$\mathrm{O}=\mathrm{C} \quad \mathrm{C}=\mathrm{O} \quad \mathrm{NC}=\mathrm{O} \mathrm{CR} \quad 3.1472 \quad 180.00$

$\mathrm{O}=\mathrm{C} \quad \mathrm{C}=\mathrm{O} \quad \mathrm{NC}=\mathrm{O} \mathrm{CR} \quad-0.073 \quad 3 \quad 0.00$

$\mathrm{O}=\mathrm{C} \quad \mathrm{C}=\mathrm{O} \quad \mathrm{NC}=\mathrm{O} \mathrm{HNCO} \quad 0.718 \quad 1 \quad 0.00$

$\mathrm{O}=\mathrm{C} \quad \mathrm{C}=\mathrm{O} \quad \mathrm{NC}=\mathrm{O} \mathrm{HNCO} \quad 2.4872 \quad 180.00$

$\mathrm{O}=\mathrm{C} \quad \mathrm{C}=\mathrm{O} \quad \mathrm{NC}=\mathrm{O}$ HNCO $\quad-0.227 \quad 3 \quad 0.00$

$\mathrm{O}=\mathrm{C} \quad \mathrm{C}=\mathrm{O} \quad \mathrm{CR} \quad \mathrm{HCMM} \quad 0.330 \quad 1 \quad 0.00$

$\mathrm{O}=\mathrm{C} \mathrm{C}=\mathrm{O}$ CR HCMM $-0.7042 \quad 180.00$

$\mathrm{O}=\mathrm{C} \quad \mathrm{C}=\mathrm{O} \quad \mathrm{CR} \quad \mathrm{HCMM} \quad 0.154 \quad 3 \quad 0.00$

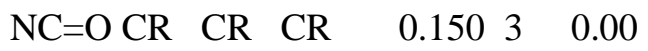

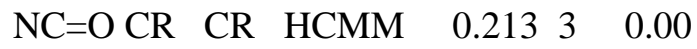

$\mathrm{NC}=\mathrm{O} C=\mathrm{O} \quad \mathrm{CR} \quad \mathrm{HCMM} \quad-0.206 \quad 1 \quad 0.00$

$\mathrm{NC}=\mathrm{O} \mathrm{C}=\mathrm{O} \quad \mathrm{CR} \quad \mathrm{HCMM} \quad 0.34622180 .00$

$\mathrm{NC}=\mathrm{O} \mathrm{C}=\mathrm{O} \quad \mathrm{CR} \quad \mathrm{HCMM} \quad 0.043 \quad 3 \quad 0.00$

$\mathrm{CR} \quad \mathrm{CR} \quad \mathrm{NC}=\mathrm{O} \mathrm{C}=\mathrm{O} \quad-0.513 \quad 1 \quad 0.00$

$\mathrm{CR} \quad \mathrm{CR} \quad \mathrm{NC}=\mathrm{O} \mathrm{C}=\mathrm{O} \quad 0.3472 \quad 180.00$

$\mathrm{CR} \quad \mathrm{CR} \quad \mathrm{NC}=\mathrm{O} \mathrm{C}=\mathrm{O} \quad 0.474 \quad 3 \quad 0.00$

$\mathrm{CR} \quad \mathrm{CR} \quad \mathrm{NC}=\mathrm{O}$ HNCO $\quad 0.276 \quad 1 \quad 0.00$

CR CR NC=O HNCO -0.19022180 .00$

$\begin{array}{lllll}\mathrm{CR} \quad \mathrm{CR} \quad \mathrm{NC}=\mathrm{O} \text { HNCO } & 0.163 & 3 & 0.00\end{array}$

$\begin{array}{lllllll}\text { CR } & \text { CR } & \text { CR } & \text { HCMM } & 0.320 & 1 & 0.00\end{array}$

$\begin{array}{llllll}\text { CR CR CR HCMM } & -0.315 & 2 & 180.00\end{array}$

$\begin{array}{llllll}\text { CR CR CR HCMM } & 0.132 & 3 & 0.00\end{array}$

$\begin{array}{llllll}\text { CR CR OR HOR } & 0.135 & 2 & 180.00\end{array}$ 
$\begin{array}{llllll}\text { CR CR OR HOR } & 0.118 & 3 & 0.00\end{array}$

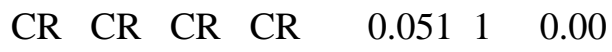

$\begin{array}{lllll}\text { CR CR CR CR } & 0.3412 & 180.00\end{array}$

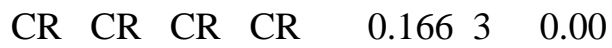

$\mathrm{CR} \quad \mathrm{NC}=\mathrm{O} \mathrm{C}=\mathrm{O} \quad \mathrm{CR} \quad 0.324 \quad 1 \quad 0.00$

CR $\mathrm{NC}=\mathrm{O} \mathrm{C}=\mathrm{O} \quad \mathrm{CR} \quad 3.0792 \quad 180.00$

CR $\mathrm{NC}=\mathrm{O} \mathrm{C}=\mathrm{O}$ CR $\quad 0.2543 \quad 0.00$

$\begin{array}{llllll}\text { CR CR OR CR } & -0.341 & 1 & 0.00\end{array}$

$\begin{array}{lllll}\text { CR CR OR CR } & 0.378 & 2 & 180.00\end{array}$

$\begin{array}{llllll}\text { CR CR OR CR } & 0.378 & 3 & 0.00\end{array}$

$\begin{array}{lllllll}\text { CR OR } & \text { CR } & \text { HCMM } & 0.285 & 1 & 0.00\end{array}$

$\begin{array}{llllll}\text { CR OR CR HCMM } & 0.160 & 2 & 180.00\end{array}$

$\begin{array}{lllllll}\text { CR OR CR HCMM } & 0.285 & 3 & 0.00\end{array}$

$\mathrm{C}=\mathrm{O} \quad \mathrm{NC}=\mathrm{O}$ CR HCMM $-1.050 \quad 1 \quad 0.00$

$\mathrm{C}=\mathrm{O} \quad \mathrm{NC}=\mathrm{O}$ CR HCMM 0.68122180 .00

$\mathrm{C}=\mathrm{O} \quad \mathrm{NC}=\mathrm{O} \quad \mathrm{CR} \quad \mathrm{HCMM} \quad 0.0113 \quad 0.00$

$\mathrm{CR} \quad \mathrm{C}=\mathrm{O} \quad \mathrm{NC}=\mathrm{O} \mathrm{HNCO} \quad-0.147 \quad 1 \quad 0.00$

$\mathrm{CR} \quad \mathrm{C}=\mathrm{O} \quad \mathrm{NC}=\mathrm{O}$ HNCO $2.9022 \quad 180.00$

$\mathrm{CR} \quad \mathrm{C}=\mathrm{O} \quad \mathrm{NC}=\mathrm{O} \mathrm{HNCO} 0.67130 .00$

$\begin{array}{llllll}\text { HCMM CR OR } & \text { HOR } & 0.298 & 1 & 0.00\end{array}$

$\begin{array}{lllll}\text { HCMM CR OR HOR } & -0.138 & 2 & 180.00\end{array}$

$\begin{array}{lllll}\text { HCMM CR OR HOR } & 0.173 & 3 & 0.00\end{array}$

$\begin{array}{llllll}\text { HCMM CR } & \text { CR } & \text { HCMM } & 0.142 & 1 & 0.00\end{array}$

$\begin{array}{llllll}\text { HCMM CR CR HCMM } & -0.693 & 2 & 180.00\end{array}$

$\begin{array}{llllll}\text { HCMM CR } & \text { CR } & \text { HCMM } & 0.157 & 3 & 0.00\end{array}$

$\begin{array}{lllll}\mathrm{HCMM} C R & \mathrm{NC}=\mathrm{O} \mathrm{HNCO} & -0.308 & 1 & 0.00\end{array}$

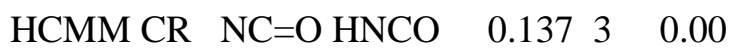

IMPROPER

$\begin{array}{lllllll}\text { CR } & \text { CR OR OR } & 0.000 & 0 & 0.00\end{array}$ 
$\begin{array}{llllll}\text { CR OR OR HCMM } & 0.000 & 0 & 0.00\end{array}$

CR CR CR NC=O $0.000 \quad 0 \quad 0.00$

CR NC=O CR HCMM $0.000 \quad 0 \quad 0.00$

$\begin{array}{lllllllll}\text { CR CR CR OR } & 0.000 & 0 & 0.00\end{array}$

$\begin{array}{lllllll}\text { CR OR CR HCMM } & 0.000 & 0 & 0.00\end{array}$

$\begin{array}{llllllll}\mathrm{CR} & \mathrm{CR} & \mathrm{OR} & \mathrm{CR} & 0.000 & 0 & 0.00\end{array}$

$\begin{array}{lllllll}\text { CR CR OR HCMM } & 0.000 & 0 & 0.00\end{array}$

$\mathrm{NC}=\mathrm{O} \mathrm{C}=\mathrm{O}$ CR $\mathrm{HNCO} \quad-1.4390000$

$\mathrm{C}=\mathrm{O} \quad \mathrm{O}=\mathrm{C} \quad \mathrm{NC}=\mathrm{O} \quad \mathrm{CR} \quad 9.284 \quad 0 \quad 0.00$

CR HCMM C=O HCMM $0.000 \quad 0 \quad 0.00$

NONBONDED nbxmod 5 atom cdiel shift vatom vdistance vswitch -

cutnb 14.0 ctofnb 12.0 ctonnb 10.0 eps 1.0 e14fac 1.0 wmin 1.5

OR $\quad 0.000000-0.152100 \quad 1.770000$

$\mathrm{O}=\mathrm{C} \quad 0.000000 \quad-0.120000 \quad 1.700000 \quad 0.000000 \quad-0.120000 \quad 1.400000$

$\mathrm{NC}=\mathrm{O} \quad 0.000000 \quad-0.200000 \quad 1.850000$

$\begin{array}{lllllll}\text { CR } & 0.000000 & -0.055000 & 2.175000 & 0.000000 & -0.010000 & 1.900000\end{array}$

$\mathrm{C}=\mathrm{O} \quad 0.000000 \quad-0.110000 \quad 2.000000$

HCMM $\quad 0.000000 \quad-0.022000 \quad 1.320000$

$\begin{array}{llll}\text { HNCO } & 0.000000 & -0.046000 & 0.224500\end{array}$

$\begin{array}{llll}\text { HOR } & 0.000000 & -0.046000 & 0.224500\end{array}$

* Made by Ratul Chowdhury, Penn State. November 2. 2018. for PNAS

* This file contains CHARMM SOLVATION PARAMTERS for GlcNAc molecule

ATOM RES RAD LAMB VOL GFREE

$\begin{array}{llllll}\text { C1 } & \text { LIG } & 2.00 & 3.5 & 14.7 & 1.00\end{array}$

O1 LIG $\quad 1.55 \quad 6.0 \quad 10.8 \quad-10.00$

$\begin{array}{llllll}\text { C2 } & \text { LIG } & 2.00 & 3.5 & 14.7 & 1.00\end{array}$

$\begin{array}{llllll}\mathrm{O} 2 & \text { LIG } & 1.55 & 6.0 & 10.8 & -10.00\end{array}$ 


\begin{tabular}{|c|c|c|c|c|c|}
\hline $\mathrm{C} 3$ & LIG & 2.00 & 3.5 & 14.7 & 1.00 \\
\hline $\mathrm{O} 3$ & LIG & 1.55 & 6.0 & 10.8 & -10.00 \\
\hline $\mathrm{C} 4$ & LIG & 2.00 & 3.5 & 14.7 & 1.00 \\
\hline $\mathrm{O} 4$ & LIG & 1.55 & 6.0 & 10.8 & -10.00 \\
\hline 7 & LIG & 2.00 & 3.5 & 14.7 & 1.00 \\
\hline O5 & LIG & 1.55 & 6.0 & 10.8 & -10.00 \\
\hline C6 & LIG & 2.00 & 3.5 & 14.7 & 1.00 \\
\hline$C 7$ & LIG & 2.00 & 3.5 & 14.7 & 1.00 \\
\hline $\mathrm{C}$ & LIG & 2.00 & 3.5 & 14.7 & 1.00 \\
\hline $\mathrm{N}$ & LIG & 1.75 & 6.0 & 11.2 & -20.00 \\
\hline $\mathrm{O}$ & LIG & 1.55 & 6.0 & 10.8 & -10.00 \\
\hline $\mathrm{H}$ & LIG & 1.00 & 3.5 & 0.0 & 0.00 \\
\hline H1 & LIG & 1.00 & 3.5 & 0.0 & 0.00 \\
\hline $\mathrm{H} 2$ & LIG & 1.00 & 3.5 & 0.0 & 0.00 \\
\hline H10 & LIG & 1.00 & 3.5 & 0.0 & 0.00 \\
\hline H11 & LIG & 1.00 & 3.5 & 0.0 & 0.00 \\
\hline H12 & LIG & 1.00 & 3.5 & 0.0 & 0.00 \\
\hline H13 & LIG & 1.00 & 3.5 & 0.0 & 0.00 \\
\hline H14 & LIG & 1.00 & 3.5 & 0.0 & 0.00 \\
\hline H3 & LIG & 1.00 & 3.5 & 0.0 & 0.00 \\
\hline $\mathrm{H} 4$ & LIG & 1.00 & 3.5 & 0.0 & 0.00 \\
\hline H5 & LIG & 1.00 & 3.5 & 0.0 & 0.00 \\
\hline H6 & LIG & 1.00 & 3.5 & 0.0 & 0.00 \\
\hline H7 & LIG & 1.00 & 3.5 & 0.0 & 0.00 \\
\hline H8 & LIG & 1.00 & 3.5 & 0.0 & 0.00 \\
\hline H9 & LIG & 1.00 & 3.5 & 0.0 & 0.00 \\
\hline
\end{tabular}

